\title{
Trends in COVID-19 Testing and Positivity Rates from a Mobile Testing Program in the Phoenix Metropolitan Area
}

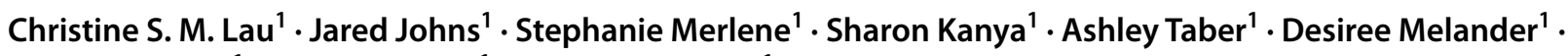 \\ Sherry Rodgers ${ }^{1} \cdot$ Pablo Prichard ${ }^{1} \cdot$ Vershalee Shukla $^{1}$
}

Accepted: 4 June 2021 / Published online: 11 June 2021

(c) The Author(s), under exclusive licence to Springer Science+Business Media, LLC, part of Springer Nature 2021

\begin{abstract}
The coronavirus (COVID-19) pandemic continues to be a global concern, with over 150 million cases worldwide. Arizona, which was recently a hotspot for COVID-19, has over 860 thousand cases. Reviewing the COVID-19 trends over time is crucial in understanding the pandemic and evaluating the impact of mitigation techniques. This current study analyzes the trends in COVID-19 testing and positivity rates from a mobile testing program in the Phoenix metropolitan area between December 2020 and April 2021. Of the 32,234 tests performed there was a total of 3654 COVID positive cases, yielding an overall positivity rate of $11.3 \%$. COVID-19 positivity rates were significantly higher in December (12.9\%) and January $(12.7 \%)$, compared to February (7.5\%), March (4.9\%), and April (6.7\%), $p<0.05$. The peak of COVID-19 cases is likely attributable to the holiday season and family gatherings, followed by a steady decline, likely due to fewer gatherings and an increase in individuals receiving the COVID-19 vaccines. Continued public health measures, including vaccinations, are critical in reducing COVID-19 transmission.
\end{abstract}

Keywords COVID-19 $\cdot$ SARS-CoV-2 $\cdot$ Mobile testing $\cdot$ Phoenix $\cdot$ Arizona

\section{Introduction}

The coronavirus (COVID-19) pandemic continues to be global public health concern. As of the end of April 2021, there were over 150 million cases and 3 million deaths worldwide [1]. In the United States alone there were over 33 million cases, with over 860 thousand cases in Arizona $[1,2]$. Arizona was once a hotspot for COVID-19 with over ten thousand new cases reported each day [3].

Several different mitigation techniques have been attempted in the State of Arizona. A state of emergency was declared on March 11, 2020 and executive orders were signed. There were capacity restrictions on restaurants and gyms, social distancing measures, and mask mandates [4]. These measures were effective in controlling the first surge of COVID-19 cases. As case counts decreased, another

Pablo Prichard and Vershalee Shukla are co-senior authors.

Christine S. M. Lau

drlau@vincerecancer.com

1 Vincere Cancer Center, 7469 E. Monte Cristo Avenue, Scottsdale, AZ 85260, USA executive order was announced in May 2020, which began re-opening businesses following strict guidelines [5]. In July 2020 , in-person schools resumed $[6,7]$. With the re-opening of the State, another increase in COVID-19 cases followed. By the end of 2020, Arizona became a hotspot with 118.3 new cases per 100,000 people [3]. In December, COVID-19 vaccines began to roll-out in Arizona, offering another line of protection to individuals.

Over a year has passed since the pandemic started, and reviewing the trends in positivity rates is important for both continued planning and mitigation of this current pandemic and future public health concerns. Social restrictions have continued to relax, and on May 12, 2021 Centers for Disease Control and Prevention (CDC) recommendations changed to permit fully vaccinated individuals to forgo social distancing and mask use in many public settings [8]. Previous loosening of guidelines in Arizona have been followed by large increases in case numbers, indicating the need for increased testing and close tracking of positivity rates as public health policies continue to evolve. This current study analyzed the trends in COVID-19 antigen positivity rates from a mobile testing site in the Phoenix metropolitan area, from December 2020 to April 2021. 


\section{Methods}

In partnership with the City of Phoenix, Vincere offered mobile COVID-19 testing in the Phoenix metropolitan area. Targeting underserved areas, different locations were selected depending on case counts, positivity rates, and the need for testing. COVID-19 antigen testing was offered at zero-cost to all individuals 12 years of age and older. Insurance was not required, and individuals without insurance were also allowed to receive testing at zero-cost. Both scheduled appointments and walk-ups were available, and testing was available 5-6 days a week, including weekend and evening hours.

Data for this was abstracted from the Vincere COVID-19 antigen testing. All data was already de-identified with no patient identifying information. Only test results (positive or negative) and date of test was included. This study was determined to be exempt by an institutional review board.

\section{COVID-19 Testing}

The Quidel SOFIA SARS Antigen Fluorescent Immunoassay, a Food and Drug Administration (FDA) Emergency Use Authorization (EUA) approved test, was used (sensitivity: $100 \%$, specificity: $98.8 \%$, positive predictive accuracy: $96.7 \%$, negative predictive accuracy: 100\%) [9]. Utilizing immunofluorescence-based lateral flow technology, this test qualitatively detects the nucleocapsid protein from SARS-CoV-2. Nasal or nasopharyngeal swabs were taken from patients, processed on-site, and results were given to the patient within 15-20 min. All tests were performed and interpreted by trained individuals, including emergency medical technicians/paramedics, medical assistants, registered nurses, and physicians.

\section{Outcome}

The primary outcome of this study was COVID-19 antigen positivity rates.

\section{Statistical Analysis}

COVID-19 antigen testing results from December 1, 2020 to April 30, 2021 was extracted. Incomplete and inconclusive data was excluded. Repeat tests on the same day were excluded. Subject data was grouped into weekly and monthly total case counts, and positivity rates were calculated. Trends were analyzed and compared to Arizona case counts and positivity rates. All statistical analysis was performed with IBM SPSS $®$ v 25 and a $p$-value of $<0.05$ was considered statistically significant.

\section{Results}

A total of 32,234 tests were performed. There was an increase in the number of tests performed in December and early January, with a steady decline afterwards. Of the tests performed, there was a total of 3654 COVID positive cases, yielding an overall positivity rate of $11.3 \%$. COVID-19 positivity rates were significantly higher in December (12.9\%) and January (12.7\%), compared to February (7.5\%), March (4.9\%), and April (6.7\%), $p<0.05$ (Table 1). Positivity rates peaked in mid-December to mid-January, followed by a steady decline to current rates at or below a positivity rate of $5 \%$ (Fig. 1).

\section{Discussion}

The COVID-19 pandemic remains a global public health issue. This study demonstrated the trends in COVID-19 testing and antigen positivity rates from a mobile COVID-19 testing program in the Phoenix metropolitan area, the largest city in Arizona.

There was an increase in COVID-19 testing and positivity rate towards the end of December 2020. Rates peaked in mid-December and mid-January before starting to decline, which mirrors the Arizona Department of Health Services (ADHS) data $[2,10]$. The rise is likely attributed to the holiday season, including Hanukkah, Christmas, and New Year's. Holidays, which were a time of family gatherings, and brought together large numbers of individuals into close contact [11]. Air travel during the holidays also increased, and airports had large crowds of passengers waiting to board flights. As per the Transportation Security Administration (TSA), over 7.1 million passengers across the country travelled through airports in the 7-days leading up to Christmas [12]. Close-contact environments, including gatherings, have demonstrated to be high risk for COVID-19 transmission [13-16]. In a study by Nishiura et al., the odds of COVID-19 transmission in closed environments were 18.7 times greater than open-air environments (OR 18.7; 95\% CI, 6.0 - 57.9) [17]. The decline in numbers following the holidays is further evidence of the risk of COVID-19 transmission among social gatherings during the holidays.

The continuous decline in COVID-19 cases and positivity trends can also be attributed to the start of COVID-19
Table 1 COVID-19 positivity rates

\begin{tabular}{llllll}
\hline & December 2020 & January 2021 & February 2021 & March 2021 & April 2021 \\
\hline Number of tests & 12,808 & 11,594 & 4329 & 1934 & 1569 \\
Antigen positivity rate $(\%)$ & 12.9 & 12.7 & 7.5 & 4.9 & 6.7 \\
\hline
\end{tabular}



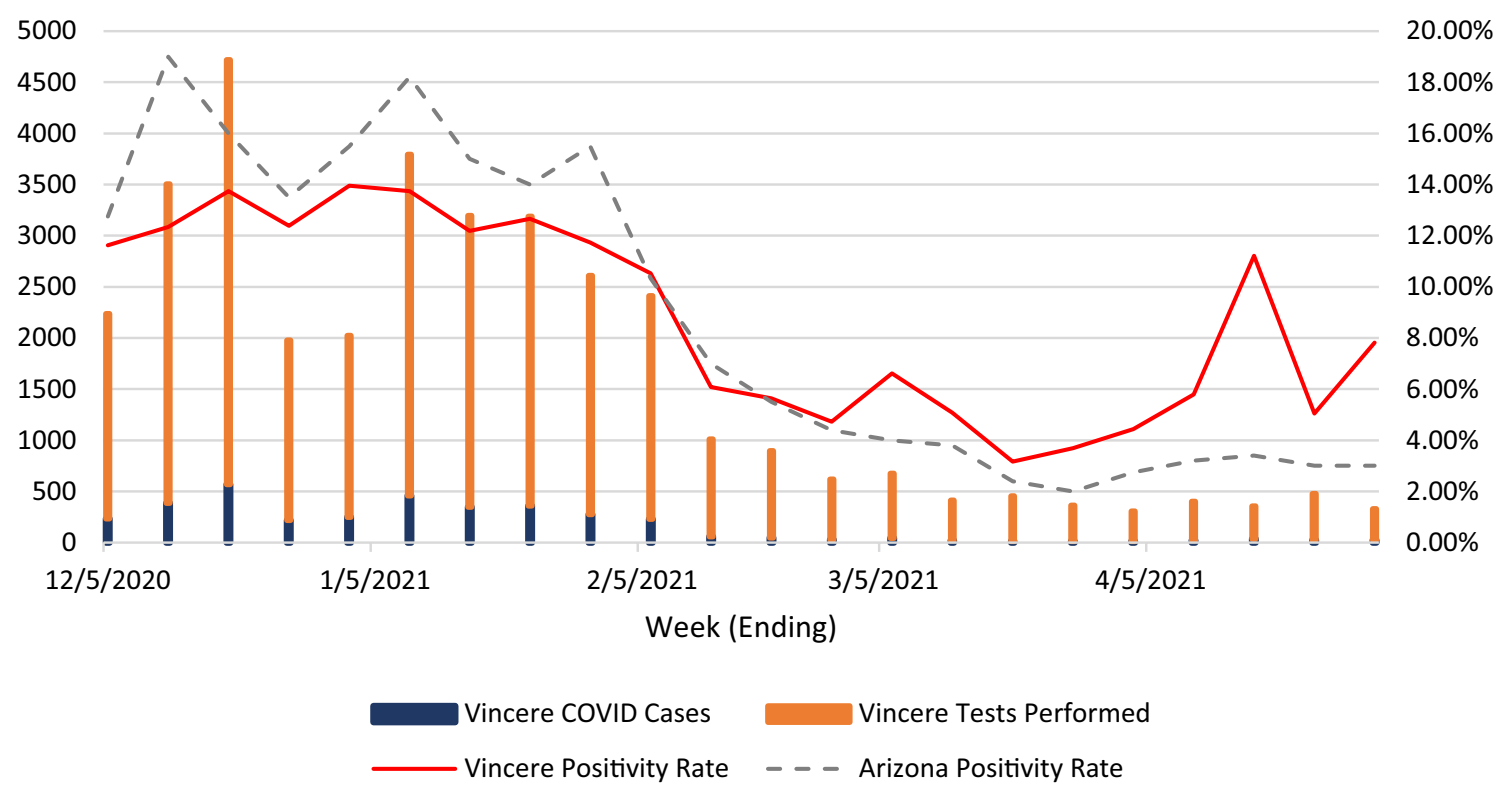

Fig. 1 Trends in COVID-19 cases and positivity rates

vaccinations. The first vaccine, Pfizer, was granted EUA on December 11, 2020, and within days, frontline workers started to get vaccinated [18]. Shortly after, the Moderna COVID-19 vaccine received EUA on December 18, 2020, and Janssen received EUA on February 27, 2021 [19, 20]. As of March 24, 2021, all individuals 16 years of age or older were eligible to receive a vaccine in Arizona, and on May 10, 2021 Pfizer's EUA was expanded to include people 12-15 years old. By the end of April 2021, 42,205 per 100,000 people in Arizona were vaccinated [21]. Approximately $40 \%$ of Arizonans received their first dose, and 32\% were fully vaccinated [21].

Until February 2021, COVID-19 positivity rates from this mobile testing site were slightly lower than Arizona reported values; however, after February 2021, positivity rates from the mobile testing site were slightly higher than Arizona rates. This may be a result of testing locations. The mobile COVID testing sites offered testing at zero-cost to all patients, regardless of insurance status. As a result, individuals were more likely to receive testing. With the roll-out of the vaccines however, these underserved areas showed lower vaccination rates compared to high-income areas [21]. The testing locations targeted underserved and low-income areas which often had larger Latino and African American populations, which have been reported to be disproportionately impacted by the pandemic. In a study by Rodriguez-Diaz et al., communities with high Latino populations ( $\geq 17.8 \%$ of the population) had higher COVID-19 cases compared to communities with lower Hispanic populations $(<17.8 \%$ of the population) in both the Northeast (adjusted relative risk
(aRR): 1.42 ; 95\% CI 1.11-1.84) and Midwest US (aRR: $1.70 ; 95 \%$ CI $1.57-1.85$ ) [22].

Vaccinations have been, and continue to be, an important step in establishing long-term immunity in a population. Administration of the COVID-19 vaccine in Maricopa County began on December 14, 2020 with limited access to the vaccines until the end of December. Vaccinations greatly increased throughout January 2021 with over 250,000 doses by January 25,2021 . Initially, vaccinations focused specifically on immunizing elderly and immunocompromised individuals as they have been shown to experience far higher instances of hospitalization and mortality due to COVID19 [2, 23]. Positivity rates at the mobile testing center and throughout Arizona were at their highest levels during this time period, and gradually decreased over subsequent weeks before lowering dramatically from 10.54 to $6.09 \%$ between the weeks of February 6 and February 13, 2021 (Fig. 1). Many individuals who received their first dose in December became fully vaccinated in February, which contributed to the substantial decrease in positivity rates. Considering the six-week period Moderna requires for full vaccination, there was a 273\% increase in cumulative doses given in Maricopa County during the relevant week of December 23-December 30, 2021 [2]. Increased immunity amongst high-risk populations coupled with improved social distancing following the Holiday season likely led to the significant decrease in COVID-19 cases and positivity rates.

Positivity rates at our testing center have been consistently higher than the Arizona average since March 2021, likely attributable in part to a focus on underserved populations. These areas are likely more susceptible to infection as 
socio-economic factors have been shown to limit the ability to practice social distancing, leading to higher COVID19 cases [24, 25]. Similar elevations in positive test results exist in the population of American Indians, who have been disproportionately affected by COVID-19 since the beginning of the pandemic [26, 27]. Positivity rates amongst this population in the Phoenix area have also been greater than average in past months and are currently at $5.3 \%$ as of 5-152021 [28]. Recent trends in positivity rates indicate that additional testing and safety precautions have the potential to be extremely valuable in these communities where incidence rates remain high. Furthermore, due to heightened vulnerability, continued testing in underserved and minority populations is crucial as social distancing and public health guidelines continue to relax.

At the end of 2020 Arizona experienced a wide-scale resurgence of COVID-19, which stressed the state's healthcare system and testing capacity. Following this, Arizona experienced a remarkable drop in positivity rates to $3 \%$ by the end of April. Given the rapidly changing conditions in the state, trends in antigen testing results can provide valuable insight into risk factors that contribute to the initial increases in disease transmission and subsequent recovery from this outbreak. Further observation and study are necessary to determine the stability of current trends due to the highly volatile spread of COVID-19 over the time period covered in this article.

Despite the results of this study, there are several limitations. The Quidel Sofia Antigen test does not differentiate between SARS-CoV and SARS-CoV-2; however, as per the $\mathrm{CDC}$, there are no current active cases of SARS-CoV-2. Furthermore, this study reports data from Phoenix metropolitan area, the largest city in Arizona, and the fourth largest city in the United States. Testing sites were selected to assist underserved communities, which had large Latino and African American populations and low-income levels. Further studies evaluating the trends among rural and remote areas are required, as access to healthcare and vaccines are more difficult in these areas.

\section{Conclusion}

Arizona COVID-19 cases and positivity rates peaked in midDecember to mid-January, likely attributable to holiday gatherings and reopening of the state. Following the holidays, case counts and positivity rates have steadily decreased. This also coincides with approximately 4-6 weeks following the start of COVID-19 vaccinations in Arizona. Evaluating trends in COVID-19 cases is crucial in understanding this pandemic and planning for this and future public health concerns. This also emphasizes the importance of targeting underserved areas, especially in ensuring testing and vaccines are available to minority and underserved areas.

Acknowledgements We would like to thank the City of Phoenix for their partnership in the COVID-19 mobile testing project. Thank-you also to all the individuals who help at the sites each day, from operational logistics to performing and processing the tests.

Funding The mobile COVID-19 testing program was done in partnership with the City of Phoenix.

\section{Declarations}

Conflict of interest The authors have no conflict of interest to declare.

\section{References}

1. Worldometer. COVID-19 Coronavirus Pandemic. Retrieved May 3, 2021 from https://www.worldometers.info/coronavirus/

2. AZDHS I COVID-19 Dashboards. Arizona Department of Health Services. Retrieved May 4, 2021 from https://www.azdhs.gov/ covid19/data/index.php

3. An interactive visualization of COVID-19/91-DIVOC. Retrieved Jan 18, 2021 from https://91-divoc.com/pages/covid-visualization/

4. Gallaway, M. S., Rigler, J., Robinson, S., et al. (2020). Trends in COVID-19 Incidence After Implementation of Mitigation Measures-Arizona, January 22-August 7, 2020. Morbidity and Mortality Weekly report, 69(40), 1460-1463. https://doi.org/10. 15585/mmwr.mm6940e3

5. Office of Governor Doug Ducey I Executive Orders. Office of the Arizona Governor. Retrieved Jan 18, 2021 from https://azgov ernor.gov/executive-orders

6. Ducey D. Executive Order 2020-2051: “Arizona Open for Learning". State of Arizona. (2020). from https://azgovernor.gov/execu tive-orders

7. Arizona Department of Health Services. Safely Returning to InPerson Instruction. (2020). Available from: https://www.azdhs. gov/documents/preparedness/epidemiology-disease-control/infec tious-disease-epidemiology/novel-coronavirus/covid-19-safelyreturn-to-in-person-instruction.pdf

8. CDC I Interim Public Health Recommendations for Fully Vaccinated People I. Retrieved May 16, 2021 from Interim Public Health Recommendations for Fully Vaccinated People I CDC

9. Quidel. Sofia SARS Antigen FIA. Product specifications. Retrieved May 4, 2021 from https://www.quidel.com/immunoassa ys/rapid-sars-tests/sofia-sars-antigen-fia

10. Johns Hopkins Coronavirus Resource Center. Arizona. Retrieved May 4, 2021 from https://coronavirus.jhu.edu/region/us/arizona

11. Lau, C. S. M., Shu, S., Mayer, J., Towns, M., Farris, A., Washington, F., Prichard, P., \& Shukla, V. (2021). COVID-19 trends in the Phoenix Metropolitan area from a mobile testing program: last quarter of 2020. Journal of Community Health, 29, 1-5. https:// doi.org/10.1007/s10900-021-00991-4

12. Transportation Security Administration. TSA checkpoint travel numbers (current year(s) versus prior year/same weekday) I Transportation Security Administration. Retrieved Jan 22, 2021 from https://www.tsa.gov/coronavirus/passenger-throughput

13. Nishiura H, Oshitani H, Kobayashi T, et al. (2020). Closed environments facilitate secondary transmission of coronavirus disease 2019 (COVID-19). medRxiv. 2020.02.28.20029272.

14. Leclerc QJ, Fuller NM, Knight LE, Funk S, Knight GM. What settings have been linked to SARS-CoV-2 transmission clusters? 
Wellcome Open Res. (2020) Retrieved Jan 9, 2021 from https:// www.ncbi.nlm.nih.gov/pmc/articles/PMC7327724/

15. Memish, Z. A., Steffen, R., White, P., et al. (2019). Mass gatherings medicine: Public health issues arising from mass gathering religious and sporting events. The Lancet, 393(10185), 2073-2084.

16. Sassano M, McKee M, Ricciardi W, Boccia S. Transmission of SARS-CoV-2 and Other Infections at Large Sports Gatherings: A Surprising Gap in Our Knowledge. Front Med. (2020) Retrieved Jan 9, 2021 from https://www.ncbi.nlm.nih.gov/pmc/articles/ PMC7273227/

17. Nishiura H, Oshitani H, Kobayashi T, Saito T, Sunagawa T, Matsui $\mathrm{T}$, et al. Closed environments facilitate secondary transmission of coronavirus disease 2019 (COVID-19). medRxiv. $2020 \mathrm{Apr}$ $16 ; 2020.02 .28 .20029272$

18. FDA I US Food and Drug Administration. Pfizer-BioNTech COVID-19 Vaccine. Retrieved May 6, 2021 from https://www. fda.gov/emergency-preparedness-and-response/coronavirus-disea se-2019-covid-19/pfizer-biontech-covid-19-vaccine

19. FDA I US Food and Drug Administration. Moderna COVID-19 Vaccine. Retrieved May 6, 2021 from https://www.fda.gov/emerg ency-preparedness-and-response/coronavirus-disease-2019-covid19/moderna-covid-19-vaccine

20. FDA I US Food and Drug Administration. Janssen COVID-19 Vaccine. Retrieved May 6, 2021 from https://www.fda.gov/emerg ency-preparedness-and-response/coronavirus-disease-2019-covid19/janssen-covid-19-vaccine

21. Maricopa County I Maricopa County COVID-19 Data. Retrieved May 4, 2021 from https://www.maricopa.gov/5671/Public-Vacci ne-Data
22. Rodriguez-Diaz, C. E., Guilamo-Ramos, V., Mena, L., et al. (2020). Risk for COVID-19 infection and death among Latinos in the United States: Examining heterogeneity in transmission dynamics. Annals of Epidemiology, 52, 46-53.e2.

23. Chen, Y., Klein, S. L., Garibaldi, B. T., et al. (2021). Aging in COVID-19: Vulnerability, immunity and intervention. Ageing Research Reviews, 65, 101205.

24. Jay, J., Bor, J., Nsoesie, E. O., et al. (2020). Neighbourhood income and physical distancing during the COVID-19 pandemic in the United States. Nature Human Behaviour, 4, 1294-1302.

25. Hawkins, R. B., Charles, E. J., \& Mehaffey, J. H. (2020). Socioeconomic status and COVID-19-related cases and fatalities. Public Health, 189, 129-134.

26. Kakol, M., Upson, D., \& Sood, A. (2021). Susceptibility of Southwestern American Indian tribes to Coronavirus disease 2019 (COVID-19). The Journal of Rural Health, 37(1), 197-199.

27. Hatcher, S. M., Agnew-Brune, C., Anderson, M., et al. (2020). COVID-19 among American Indian and Alaska native persons-23 States, January 31-July 3, 2020. Morbidity and Mortality Weekly Report, 69(34), 1166-1169.

28. U.S. Department of Health and Human Services I "Indian Health Service (IHS)." I Coronavirus I cited [16 May 2021]. Available from: www.ihs.gov/coronavirus/

Publisher's Note Springer Nature remains neutral with regard to jurisdictional claims in published maps and institutional affiliations. 